\title{
Knowledge and attitudes of Korean parents towards their son's circumcision: a nationwide questionnaire study
}

\author{
S.-J. OH, K.D. KIM ${ }^{1}$, K.M. KIM, K.S. KIM ${ }^{2}$, K.K. KIM ${ }^{3}$, J.S. KIM ${ }^{4}$, H.G. KIM ${ }^{5}$, Y.N. WOO ${ }^{6}$, Y.L. YOON ${ }^{7}$, \\ S.D. $\mathrm{LEE}^{8}$, S.W. HAN ${ }^{9}$, S.I. $\mathrm{LEE}^{10}$ and H. CHOI \\ Department of Urology, College of Medicine, Seoul National University, ${ }^{1}$ Chung-Ang University, ${ }^{2}$ Keimyung University, \\ ${ }^{3}$ Hallym University, ${ }^{4}$ Wonkwang University, ${ }^{5}$ Asan Foundation Kangnung Hospital, ${ }^{6}$ Hanyang University, \\ ${ }^{7}$ Chungnam University, ${ }^{8}$ Pusan National University, ${ }^{9}$ Yonsei University, and ${ }^{10}$ Department of Preventive Medicine, \\ College of Medicine, Ulsan University, Seoul, Korea
}

Objective To evaluate knowledge about the foreskin and circumcision, and to understand the attitudes of parents to circumcision in Korea, where circumcision in childhood is widely practised with no particular religious or medical background.

Subjects and methods A nationwide study involving questionnaires was conducted on 5500 parents with at least one son attending elementary school. Responses were obtained from one of the parents.

Results The response rate was 76.1\% (4183); circumcision was most common in boys when aged 11 years, followed by neonatal circumcision. Of the parents, 91.3\% believed that circumcision is necessary, while $2.1 \%$ believed it to be unnecessary. The principal reasons given for circumcision were 'to improve penile hygiene' $(82.4 \%)$, followed by 'to improve future sexual potency' $(7.5 \%)$. Among those who did not believe circumcision to be necessary, the most common reason was the expectation of spontaneous retraction of the prepuce with age (55.1\%). Most $(88.4 \%)$ of the parents believed that smegma is not a clean material, and is infected by microorganisms. Most parents $(80.6 \%)$ thought that circumcision would prevent genital tract infection of the future spouse. Peer pressure was one of the most influential factors in deciding upon circumcision; $41.9 \%$ of the parents were anxious that their child might be ridiculed by his peer group unless he was circumcised, while $27.4 \%$ of the parents believed that their child might be ridiculed if he was circumcised. Mothers were more positive about circumcision than fathers $(P<0.05)$. Parents with a higher education and higher socio-economic status were also more positive about circumcision $(P<0.05)$. Mothers were prone to emphasize improved sexual potency $(P<0.05)$. There was no significant difference in response between urban and rural areas.

Conclusion This study indicates that common beliefs held by parents about the prepuce or circumcision differ significantly from current medical knowledge, and these beliefs have a major influence on the practice of circumcision in Korea. More clinical research on the natural history of the foreskin is needed, and it is critical that both children and parents are informed about the potential benefits and disadvantages of circumcision.

Keywords circumcision, Korean, prepuce, foreskin

\section{Introduction}

Circumcision has been used as a religious marker in Moslem and Jewish communities; in the USA, neonatal circumcision for nonreligious reasons prevails. Meanwhile, circumcision is also widely practised among young adolescents in Korea, with no religious background. No other operation is performed as often in Korea, or on the basis of such poor understanding.

Traditionally, Korea has been under the strong influence of Confucianism and Buddhism. Circumcision

Accepted for publication 8 December 2001 is not widely practised in neighbouring Asian countries, e.g. China, Japan, and Taiwan, or even in North Korea, which shares the same cultural background. Throughout a long history, it is evident that Koreans did not practise circumcision until the end of World War II or the Korean War. However, since 1945, Korea has been geographically and strategically influenced by the USA. Although the practice of circumcision seems to have started in Korea having been introduced by Americans, it is now deeply incorporated into present Korean culture [1]. Nevertheless, even today, there is little scientific knowledge to explain the practice of circumcision in Korea. 
Routine circumcision has ethical, medical, economic and psychological significance for children. In Korea, circumcision is mostly applied in elementary-school children [1]. Naturally, most circumcisions are decided upon by parents, and therefore the general beliefs held by parents govern the practice in Korea. To date, very little is known about parents' attitudes toward a child's circumcision. The aim of the present study was to evaluate the opinion or general knowledge of Korean parents on circumcision.

\section{Subjects and methods}

This study was carried out by 11 members of the Korean Society of Paediatric Urology (KSPU). Each KSPU member visited a designated elementary school and distributed 500 questionnaires. The five major geographical regions of South Korea were incorporated into the planned survey, and the elementary schools in both rural and urban areas were chosen. A total of 5500 questionnaires was distributed via teachers to the boys in the third (10-year-old) to sixth grades of each school. The questionnaires were distributed to each boy, and only one questionnaire was reviewed and answered by one of the parents. The questionnaires were completed anonymously. The boys returned the completed questionnaires to their teachers, and these were collected by the KSPU staff for database processing and statistical analysis. The survey was distributed in November 1999 and the last response was received during January 2000.

The questionnaire items were developed from general medical knowledge about circumcision, and consisted of 12 questions that addressed demographic details. The age of circumcision was determined in a circumcised population. Questions probed parents' concerns about circumcision, and the necessity and reasons for circumcision. If parents did not believe in the need for circumcision, specific reasons were requested. Parents were also asked for their opinions about the potential benefits and disadvantages of circumcision. The relationship between circumcision and genital hygiene, infectious diseases and sexual potency was also questioned, as was the level of peer pressure about circumcision.

Questions were presented in multiple-choice format, 'yes/no' format or the parents were asked to complete the blank option. Other variables included age, sex, level of education, socio-economic status and religion. A rural area was defined as one with a county-based local government.

Preliminary pre-testing was conducted on a group of 50 parents to ensure that the questionnaire items were valid and provided reliable answers. Some of the questionnaire items were modified by the feedback obtained. The layout of the print and the arrangement of the questionnaire were designed to allow it to be easily read and answered by the parents. An epidemiologist (S.I.L) supervised all of the procedures mentioned above.

For analysis, descriptive ratings were used for individual questionnaire items. Relationships among variables were examined using the chi-square test and Pearson's correlation coefficient, with $\mathrm{P}<0.05$ considered to indicate statistical significance.

\section{Results}

The response rate was $76.1 \%(4183 / 5500)$; all incomplete (542) and unqualified responses (49) were excluded, giving a total of 3592 responses that were finally analysed. Fathers accounted for slightly over half the respondents, with mean (SD) ages of 40.6 (4.0) and 37.5 (3.4) years for fathers and mothers, respectively (Table 1). The distribution of their offspring are also shown in Table 1. Most of the responses (84.2\%) were from urban rather than rural residents.

The responses concerned 5096 boys, including brothers of the original student; 2102 were circumcised and among these the circumcision was carried out preferentially at two ages, being most frequent at $\approx 11$ years, followed by neonatal (Fig. 1).

Of the 3592 parents questioned, $96.7 \%$ were concerned about the circumcision of their son; $>91 \%$ believed that

Table 1 The demographic details of the 3592 responders

\begin{tabular}{lc}
\hline Characteristic & \% of responders \\
\hline Respondents & \\
$\quad$ Father & 52.2 \\
Mother & 47.8 \\
Responder's children & \\
1 son + daughter & 38.5 \\
2 sons & 34.8 \\
1 son & 12.0 \\
Others & 14.7 \\
Urban & 84.2 \\
Rural & 15.8 \\
Religion & \\
Buddhism & 30.1 \\
Protestant & 27.1 \\
Catholic & 11.0 \\
Others & 1.5 \\
None & 30.3 \\
Education & \\
Less than high school & 11.0 \\
High school graduate & 42.8 \\
More than college graduate & 46.2 \\
Family income (Won, monthly) & \\
$<1000000$ (US \$770) & 13.5 \\
1 000 001-2000 000 & 40.8 \\
> 000 001-3000 000 & 26.2 \\
&
\end{tabular}




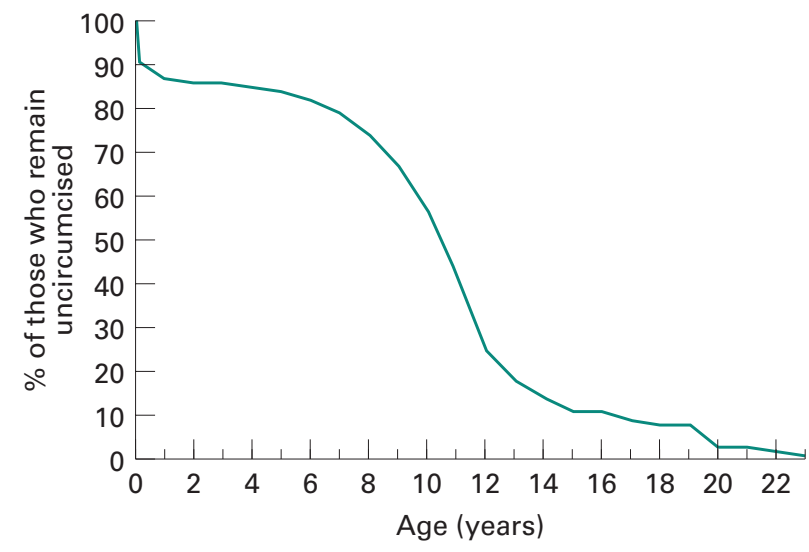

Fig. 1. The age of circumcision among children for whom information was provided by the parents.

circumcision was necessary (36.2\% absolutely necessary and $55.1 \%$ probably necessary), while $2.1 \%$ believed it to be unnecessary (with 6.6\% 'don't know'). Among those who believed circumcision was necessary, $84.7 \%$ stated that they had specific reasons for circumcision, while $15.3 \%$ said they had no particular reasons.

The major reasons given by parents for having their sons circumcised and their opinion of the best age for the procedure are summarized in Table 2. Among those who considered circumcision unnecessary, the principal reasons are also shown in Table 2. Most parents (88.4\%) believe that smegma in the preputial sac is not a clean material, and is infected by microorganisms; 1455 (40.5\%) parents observed that their child had erythema or pain of the prepuce when uncircumcised.

The expected benefits of circumcision as believed by the parents are also shown in Table 2. Over the half the parents believed that circumcision may help prevent bladder or kidney infection, prevent cervical cancer and genital tract infection of the future spouse, improve sexual potency, prevents premature ejaculation and have a good effect on penile growth.

In all, 583 parents $(16.2 \%)$ did not give consent for their son to be circumcised during the neonatal period. The major reasons are shown in Table 2; however, $34.9 \%$ of the parents thought that anaesthesia is unnecessary during neonatal circumcision because neonates do not feel pain.

Peer pressure was one of the strongest influencing factors when deciding upon circumcision: 1505 (41.9\%) of parents are anxious that their child might be ridiculed by his peer group unless circumcised, while 984 (27.4\%) parents thought that their child might be ridiculed if circumcised.

When asked whether parents had ever thought of circumcising their son(s), mothers, better educated parents and parents with a higher socio-economic status (all
Table 2 The reasons given for circumcision among parents who responded that the circumcision of their son(s) is necessary or unnecessary, the perceived benefits, the opinion of parents about peer pressure, and reasons for disagreeing with neonatal circumcision

\begin{tabular}{lc}
\hline Questionnaire items & Response, \% \\
\hline Reasons for circumcising & \\
Better penile hygiene & 82.4 \\
Improvement of future sexual function & 7.5 \\
So that the child looks like his peers & 1.1 \\
Religious reason & 0.3 \\
Better appearance & 0.2 \\
Other reasons & 8.5 \\
Best age for childhood circumcision & \\
Late elementary school & 42.2 \\
Early elementary school & 26.1 \\
Neonatal & 18.0 \\
Other & 13.7 \\
Reasons for not circumcising & \\
Prepuce spontaneously retracts with age & 55.1 \\
Do not know exact medical benefits & 20.3 \\
Afraid of postoperative complication & 2.9 \\
Other & 21.7 \\
Believed benefits of circumcision & \\
Prevention of: & \\
$\quad$ penile cancer & \\
bladder or kidney infection & \\
$\quad$ cervical cancer of the future spouse & \\
genital tract infection of the future spouse & 85.7 \\
Improvement of sexual potency & 53.4 \\
Prevention of premature ejaculation & 64.7 \\
Better growth of phallus & 62.6 \\
Better urinary stream & 60.7 \\
Reasons for not agreeing to neonatal circumcision & \\
Pain & 54.2 \\
Bad cosmesis & \\
Ridiculed by peers & 22.8 \\
Risky & \\
Other & \\
\hline & \\
\hline
\end{tabular}

$P=0.001)$ were more likely to give affirmative responses. Mothers rather than fathers felt more positively that circumcision is necessary $(P<0.05)$.

Although the major reason for circumcision was improved penile hygiene and improved sexual potency, in that order, this depended upon the gender of the parent. A significantly higher proportion of mothers (11.9\%) regarded the major reason for circumcision to be improved sexual potency (vs fathers, 4.6\%; $P<0.05$ ). Parents with higher education or higher socio-economic status stressed penile hygiene rather than sexuality $(P<0.05)$.

Parents with higher education or higher socioeconomic status were more likely to believe that smegma is not a clean material $(P<0.05)$ and that circumcision improves penile hygiene $(P<0.05)$. However, 
parent gender was not a significant factor for smegma or penile hygiene $(P>0.05)$. Geographical location, rural/urban area and religion did not significantly affect the responses to the questionnaire items $(P>0.05)$.

\section{Discussion}

Routine male circumcision is widespread in Korea but the background differs considerably from those in North America or the Middle East. Surprisingly, the culture of circumcision in Korea is not well understood [1,2]. The present study is the first to survey parents' opinions about circumcision in Korea. We consider that the general knowledge and attitudes of the parents are important, because most circumcision is performed upon school children. Before starting this survey, questionnaire items that interest or concern most Koreans were selected. Through the preliminary survey, some items were subsequently added and others modified. Therefore, the general concepts and beliefs affecting circumcision, as held by Korean parents, are included in this survey.

There is a common core of knowledge on circumcision in diverse cultures worldwide, but Korean circumcision has several unique characteristics, the most peculiar of which is that most boys are circumcised in early adolescence, not neonatally, as in America (Fig. 1); the responses from the parents support this result. Most parents believed that the best time for circumcision is during late elementary school or slightly earlier. Perhaps some parents $(34.9 \%)$ preferred neonatal circumcision because they believe that neonates are unable to feel the pain of circumcision, although the main reason given by parents not consenting to neonatal circumcision was that neonates feel the pain of circumcision (42.8\%), suggesting that some parents do believe that pain is a disincentive. Although general anaesthesia is used worldwide for neonatal circumcision, local anaesthesia is more practical. Thus the earliest a child can tolerate local anaesthesia and be circumcised as an outpatient is during early elementary school, and perhaps this is why parents choose late elementary school as the best time for circumcision. Another reason for this timing is that parents tend to consider circumcision as a 'rite of passage' into adulthood, because their sons are just beginning adolescence. This may be related to the expectation of an improvement in sexual function, as suggested by the survey result.

One of the unique features of the beliefs held by Korean parents is that a major reason in parental decisions about circumcision is an improvement in penile hygiene (82.4\%); most parents (88.4\%) agreed that smegma is a material that is infected with microorganisms, and thus most believe that unclean material covered by the prepuce may cause infections of the genital organs of the future spouse. Consequently they believe that penile hygiene is improved by removing the prepuce, thus preventing the accumulation of smegma. This belief is fortified by the experience of some parents (40.5\%) that their son(s) had redness or pain of the prepuce, an experience which might have led the parents to believe that smegma within the uncircumcised foreskin could be related to the inflammation of the phallus. The negative attitude to smegma is further supported by the response that the reason circumcision is unnecessary is that 'the foreskin spontaneously retracts with age'; this reaction was obtained from over the half the parents who believed that circumcision is unnecessary. 'Spontaneously retracted with age' is a unique concept in Korea, as Kim et al. [1] explained in detail. Briefly, Korean men widely consider this retraction as being 'naturally circumcised', indicating that the phallus may be similar to the shape of a circumcised phallus. This expectation may be partly related to the lifelong penile hygiene practices adopted by some uncircumcised men. Analysis of the present data showed that parents of well-educated or higher socio-economic status were significantly more likely to believe that smegma is not a clean material. In addition, these parents were also significantly more likely to believe that the reason for circumcision is improved penile hygiene. Although there is anecdotal information on the natural history of the prepuce [3] and the nature of smegma, it is still largely insufficient.

For the medical benefits of circumcision, most parents believe that circumcision prevents inflammatory disease and pelvic cystitis/pyelonephritis of the male, and cervical cancer in the future spouse. The medical benefits of circumcision for genitourinary infection are controversial. Some protective effect of circumcision against UTI during the first year of life was proposed by Wiswell et al. [4] but this awaits confirmation in a controlled prospective trial. The inconsistency of the results and the number of confounding factors make it impossible to link the foreskin with UTI; such infections may provide a theoretical indication for circumcision, but there is no firm evidence to support this belief. In addition, the medical literature does not support the theory that circumcision prevents sexually transmitted diseases. In Korea, most parents do not cite this belief as a general medical reason; rather, Korean parents regard smegma as unclean material that may cause inflammation, as mentioned.

As perceived by Korean parents the other major benefits of circumcision are improved sexual potency, penile growth, and the prevention of premature ejaculation; mothers, rather than fathers, believe these to be true. Some parents believe that circumcision reduces male sensitivity and coital enjoyment, while others argue 
that circumcision may afford greater ejaculatory control $[5,6]$. Recent reports suggest that the sensitivity of the circumcised glans may be reduced [7-9] but there is little consensus about the role of the foreskin in sexual performance and satisfaction.

The investigation of the psychological and social effects of circumcision in the present study provide a valuable new area of inquiry. Social institutions are important in the development and continuance of circumcision in Korea; peer pressure was one of the most influential factors in deciding to circumcise. Mass psychology may be important in both parents and children in Korea in this respect. A significant proportion of the parents who believed that circumcision is necessary (15.3\%) had no firm conviction to support the need and gave no specific reason for circumcising their sons, perhaps because they felt their reasons were not significant enough or they felt more comfortable if they followed the cultural norm. In addition, boys also want to follow the general practice; $42 \%$ of the parents were anxious that their son(s) might be ridiculed by peers unless they were circumcised, while $27 \%$ of parents believed that their child might be ridiculed if he was circumcised. Significantly many parents simply feared that their children might be disadvantaged among their peers. However, there are opposing opinions about the effect of peer pressure on the decision to circumcise. The reason parents believe their child might be ridiculed is that he might be circumcised earlier than his peers. Many parents replied that their son(s) might be ridiculed by peers unless he was circumcised, because peers boast of having superior penises because of circumcision. To the child, cultural identity or the sense of belonging to a group is of greater significance than a minor invasive treatment. Cultural conformity is a major force which perpetuates circumcision for other than religious reasons.

Parents' attitudes on circumcision varied significantly, affected by several factors which included the level of education and the gender of the parent. Mothers were more likely to be concerned about a child's circumcision and have a more positive attitude towards circumcision than were fathers. Significantly, more mothers expected improved sexual potency. Korean fathers generally appeared to take less interest in the decision to circumcise. The reasons mothers gave for deciding to have their sons circumcised suggest that the decision was often irrational. Those who were better educated or had a higher socio-economic status believed that the benefits of circumcision were improved penile hygiene rather than improved sexuality. Thus there may be significant social variation in the beliefs held about circumcision. Better educated parents were more likely to be exposed to any prevailing scientific wisdom favouring circumcision, and more like to be exposed to significant social pressures to conform to this wisdom than were less educated parents. However, Koreans of various religions or from geographical regions showed no significant differences in their attitudes to circumcision, suggesting a uniformity of attitude towards circumcision in Korea.

The awareness of circumcision is changing but almost all responders had insufficient information about circumcision. In Korea more boys are circumcised during elementary school than neonatally, which differentiates Korea from other regions where circumcision is common. If parents have detailed information about the advantages and disadvantages of circumcision they may pass the information to their sons before deciding to circumcise. Removing the normal genital anatomy in children and infants should be deferred until an individual can make an informed decision. Parents should be fully informed by qualified medical personnel about the merits and demerits of the procedure. The present study indicates an increasing need for medical practitioners to provide adequate and accurate information about circumcision.

In conclusion, common beliefs held by parents about the prepuce or circumcision differ significantly from current medical thinking, and these beliefs have a major influence on the practice of circumcision in Korea. Children and parents must be educated about the potential benefits and disadvantages of circumcision.

\section{Acknowledgements}

We thank all of the teachers and the parents who participated in this study, Ferring Pharmaceuticals Korea Ltd. who supported this survey, and Seung-Yup Oh who provided assistance by managing the database.

\section{References}

1 Kim DS, Lee JY, Pang MG. Male circumcision: a South Korean perspective. BJU Int 1999; 83 (Suppl.): 28-33

2 Pienkos EJ. Circumcision at the 121st Evacuation Hospital. Report of a questionnaire with cross-cultural observations. Military Med 1989; 154: 169-71

3 Kayaba H, Tamura H, Kitajima S, Fujiwara Y, Kato T, Kato T. Analysis of shape and retractability of the prepuce in 603 Japanese boys. J Urol 1996; 156: 1813-5

4 Wiswell TE, Geschke DW. Risks from circumcision during the first month of life compared with those for uncircumcised boys. Pediatrics 1989; 83: 1011-5

5 Morgan W. Penile plunder. Med J Aust 1967; 1: 1102-3

6 Burger R, Guthrie T. Why circumcision? Pediatrics 1974; 54: 362-4

7 Preston EN. Whither the foreskin? A consideration of routine circumcision. JAMA 1970; 213: 1853-8

8 Cleary T, Kohl S. Overwhelming infection with group B beta-hemolytic streptococcus associated with circumcision. Pediatrics 1979; 64: 301-3 
9 Immerman RS, Mackey WC. A biocultural analysis of circumcision. Soc Biol 1997; 44: 265-75

\section{Authors}

S.-J. Oh, MD, Assistant Professor.

K.D. Kim, MD, Professor.

K.M. Kim, MD, Professor.

K.S. Kim, MD, Professor.

K.K. Kim, MD, Professor.

J.S. Kim, MD, Assistant Professor.

H.G. Kim, MD, Assistant Professor.

Y.N. Woo, MD, Professor.

Y.L. Yoon, MD, Professor.

S.D. Lee, MD, Assistant Professor.

S.W. Han, MD, Associate Professor.

S.I. Lee, MD, Associate Professor.

H. Choi, MD, Professor.

Correspondence: Hwang Choi, Department of Urology, Seoul National University Hospital, 28 Yongon-Dong, Chongno-Gu, Seoul 110-744, Korea.

e-mail: hchoi@snu.ac.kr

\section{Appendix}

\section{The questionnaire}

Gender of the responder: (male, female)

Age: ( ) years old

Number of children you have: male ( ), female ( )

Age of your son: First son ( ) years old; Second son ( ) years old; Third son ( ) years old.

Whether your son underwent the circumcision:

First son (done, not done); Second son (done, not done); Third son (done, not done).

The age at which your son(s) underwent circumcision(s):

First son ( ) years old; Second son ( ) years old; Third son ( ) years old.

\section{Questions:}

Q1. Have you ever thought of the circumcision of your $\operatorname{son}(\mathrm{s})$ ?

(1) Yes. (2) No.

Q2. Do you think circumcision is necessary for your son(s)? (Choose one of the following)

(1) Absolutely necessary. Go to Q3-1

(2) May be necessary. Go to Q3-1

(3) I do not know. Go to Q3-1

(4) May not be necessary. Go to Q4

(5) Absolutely unnecessary. Go to Q4

Q3-1. Do you have any particular reason for viewing circumcision to be necessary?

(1) Yes. (2) No.

Q3-2. What is the main reason for circumcision? (Choose one)

(1) Better penile hygiene

(2) To help improve sexual function as an adult

(3) Good appearance (cosmetic reason)

(4) Look like his peers (peer pressure)

(5) Religious reasons

(6) Others (Briefly state why)
Q3-3. When do you think it is the best time to have your son circumcised? (Choose one of the following)

(1) Immediately after birth

(2) Pre-school period

(3) Early elementary school period

(4) Late elementary school period

(5) Middle school period

(6) High school period

(7) Just before military recruitment

(8) During military service

(9) Just before marriage

(10) Middle age

(11) Old age

(12) I have never given a thought. Go to Q5

Q4. If you think circumcision is unnecessary, what is the main reason? (Choose one of the following)

(1) Because I do not know the exact medical benefits.

(2) Because I am anxious about postoperative complications.

(3) Because the peers may ridicule the appearance of circumcised phallus.

(4) Because it may spontaneously be retracted with time.

(5) Others (Briefly state why).

Q5. Please mark $\mathrm{O}$ if you agree, mark $\mathrm{X}$ if you do not agree.

(A) The white material under uncircumcised preputial skin is an unhealthy material infected by microorganisms. ( )

(B) Circumcision may help prevent penile cancer. ( )

(C) Circumcision may help prevent bladder or kidney infection. ( )

(D) Circumcision may help prevent cervix cancer of the sexual partner. ( )

(E) Circumcision may help prevent genital tract infection of the sexual partner. ( )

(F) My son has experienced inflammation, redness and pain of the penile prepuce when uncircumcised. ( )

Q6. Have you ever had your newborn son circumcised within the first month?

(1) Yes. (2) No.

Q7. What was your main reason for disagreeing with neonatal circumcision? (Choose one of the following)

(1) My son may be ridiculed by the appearance of the circumcised phallus.

(2) It may be risky because the penis of a newborn is too small to circumcise.

(3) It looks bad when done too early.

(4) Even a newborn may feel the pain from circumcision.

(5) I have heard from others that there are many complications.

(6) Others (Please specify).

Q8. Please mark $\mathrm{O}$ if you agree, mark $\mathrm{X}$ if you do not agree.

(A) My son may be ridiculed by peers with the appearance of circumcised phallus if uncircumcised. ( )

(B) My son may be ridiculed by peers if he remains uncircumcised. ( )

(C) Anaesthesia is unnecessary in neonatal circumcision because neonates do not feel pain. ( ) 
432 S.-J. OH et al.

(D) Circumcision improves male sexual potency in adulthood. ( )

(E) Circumcision effectively prevents premature ejaculation by decreasing penile sensitivity. ( )

(F) Circumcision facilitates penile growth. ( )

(G) Circumcision strengthens the urinary stream. ( )

Q9. What is your level of education?
(1) No education
(2) Elementary school
(3) Middle school
(4) High school
(5) College or University

Q10. How much is the overall monthly income of your family? [List of incomes not detailed here]

Q11. What is your religion?

(1) Buddhism

(2) Protestant

(3) Catholic

(4) Other

(5) I have no religion.

Q12. Where do you live? [List of locations not detailed here] 